\title{
Single-ended method to accurately calculate UHV AC transmission line frequency parameters
}

\author{
GAN Fan ${ }^{1}$, ZHANG Nana ${ }^{1}$, LI Yun-ge ${ }^{2}$,Fu Zhouxing ${ }^{1}$ \\ ${ }^{1}$ School of Electrical and Control Engineering,Xi'an University of Science and Technology,Yanta District,Xi'an, China \\ ${ }^{2}$ Electric Power Research Center,State Grid Shaanxi Electric Power Research Institute, Hangtian,Xi'an, China
}

\begin{abstract}
The power frequency parameter of transmission line is the basic parameter of line over-voltage calculation. It is necessary to get the parameters of $1000 \mathrm{kV}$ AC double-circuit line on the same tower in order to guide the operation of the project. In this paper, a method of accurately calculating UHV transmission line parameters is proposed. The length of the line is known, the voltage and current at the head of the line are measured, and the zero sequence, positive sequence total impedance and total capacitive reactance are obtained. Based on the distribution parameters Calculation formula, the line unit capacitance, resistance and inductance parameters can be obtained. The method of this paper is compared with the simulation of transmission line parameters in ATP simulation. And Through the actual measurement of the frequency parameters of Yu-heng $1000 \mathrm{kV}$ transmission line, it is verified that this method is suitable for the calculation of distribution parameters of UHV transmission lines.
\end{abstract}

\section{INTRODUCTION}

UHV is the world's advanced transmission technology, with the advantages of large transmission capacity, long transmission distance, low line loss and less occupied land. The uneven distribution of power resources and load center in our country makes the research of UHV transmission technology more and more important. The distribution characteristics of UHV transmission lines determine the basic characteristics of transmission lines. Accurate positive sequence zero sequence parameters are important data in fault analysis, network loss, short circuit, relay setting and power flow calculation. Therefore, UHV transmission Line Power frequency-sequence parameters for the system engineering is very important, these parameters can be obtained by actual measurement and calculation.

Literature [1] through a large number of test data and waveform, the Power frequency-sequence parameters of 750kV Pingliang - Qianxian double-circuit line on the same tower were corrected, and obtained the work frequency sequence parameter and the phase parameter. Literature [2-3] measured the voltage at the head of the line, current and power, the use of mathematical relationship between the three tests to calculate the line Power frequency-sequence parameters. The single-ended measurement method proposed in Literature [4] is to use the equivalent calculation to approximate the result of the power frequency parameter. Literature [5] mainly uses the two-terminal synchronous measurement technology based on GPS to measure the zero-sequence impedance parameters of multiple circuits on the same tower and does not involve the measurement of parameters such as positive sequence parameters and zero sequence capacitance. In engineering, a convenient conventional approximation method is used, that is, the total impedance and total capacitance of the line are obtained by measuring the voltage and current, and dividing the total impedance and the total capacitive reactance by the length of the line to obtain the line frequency parameters of the line.

In summary, line frequency sequence parameters offline measurement methods commonly used conventional approximation method, single-ended method, double-ended synchronous measurement method. The conventional approximation method is simple and convenient to calculate, but the error is larger when it is used for long lines. The existing single-end method has more measurements, and the calculation uses Maclaurin approximation, which results in inaccurate calculation. The double-ended method needs to measure the voltage and the current at both ends of the line synchronously, which increases the complexity of operation and calculation. This paper presents a single-ended method to accurately calculate the power frequency parameters of UHV AC transmission line: measuring the voltage and current at the head of the UHV AC transmission line, and analyzing and calculating the relationship between unit resistance $\mathrm{R}_{0}$, unit inductance $\mathrm{L}_{0}$, unit capacitance $\mathrm{C}_{0}$ and unit conductance $G_{0}$ in the distribution parameters of UHV AC transmission lines ,by using the long-line equation to get the corresponding transmission line parameter formula, so as to get enough accurate frequency parameters. The calculated results are compared with the results of ATP calculation. The method is applied to obtain

\footnotetext{
*Corresponding author: 285103986@qq.com,1311988379@qq.com
} 
the required frequency and frequency parameters of Yu-Heng I and II lines of UHV double-circuit lines on the same tower. The result of the actual project can be got.

\section{Uhv Transmission Line Calculation}

It is often assumed in theoretical study that the circuit model is composed of lumped parameter elements. However, in the actual circuit, the electromagnetic phenomena tend to be distributed, so it has some limitations to establish a centralized parameter model, which need to refer to the distribution parameter model. Set the transmission line length 1 , when $1>200 \mathrm{~km}$ will be treated according to the distribution parameters, namely the long-line model.

It is assumed that the overhead line is a uniform transmission line, and the resistor $\mathrm{R}_{0}$, the inductor $\mathrm{L}_{0}$, the capacitor $\mathrm{C}_{0}$, and the conductance $\mathrm{G}_{0}$ are unit parameters of the transmission line. In this paper, the sinusoidal steady-state analysis of the uniform transmission line equation is used. The voltage and current at the beginning and the end of the transmission line have the following relationship (called the long-term equation):

$$
\left[\begin{array}{c}
U_{1} \\
I_{1}
\end{array}\right]=\left[\begin{array}{cc}
\operatorname{ch} \gamma l & Z_{C} \operatorname{sh} \gamma l \\
\operatorname{sh} \gamma l / Z_{c} & \operatorname{ch} \gamma l
\end{array}\right]\left[\begin{array}{c}
U_{2} \\
I_{2}
\end{array}\right]
$$

among them,

$$
\begin{gathered}
Z_{C}=\sqrt{\frac{R_{0}+j \omega L_{0}}{G_{0}+j \omega C_{0}}} \\
\gamma=\sqrt{\left(R_{0}+j \omega L_{0}\right) \cdot\left(G_{0}+j \omega C_{0}\right)}
\end{gathered}
$$

Where: $\dot{U}_{1}, \dot{I}_{1}$ is the voltage and current at the head of the line; $\dot{U}_{2}, \dot{I}_{2}$ is the voltage and current at the end of the line; $Z_{C}$ is the wave impedance of the transmission line; $\gamma$ is the propagation constant of the transmission line; $l$ is the length of the transmission line. The conductance $G$ is often ignored for simplicity of calculation.

The basic principle of the method presented in this paper is as follows: Knowing the value of the voltage and current at the head of a transmission line with a length of $l$, we can calculate $X_{c}$ and $Z_{X}$, and its impedance angle $\alpha$. By using the terminal voltage $U_{2}=0 \mathrm{~V}$ at the end of a line, and the terminal current $I_{2}=0 \mathrm{~A}$ when the line is open at the end of the line, a set of equations with unknown $\gamma$ and $Z_{C}$ can be listed. The equations are solved for $\gamma$ and $Z_{C}$, then the power frequency parameters $R_{0}, L_{0}, C_{0}, G_{0}$ of the transmission line that we need are obtained by equations (2) and (3). The specific process is as follows:

Suppose the total capacitance of the line, the total impedance is as follows:

$$
\begin{aligned}
& X_{C}=-j \frac{1}{\omega C} \\
& Z_{X}=R+j \omega L
\end{aligned}
$$

Where, $R$ is the total resistance of the transmission line, $L$ is the total inductance of the transmission line, $C$ is the total transmission line capacitance, $\omega$ is the angular frequency of the line.

Because of short circuit at the end of the line,, then equation (1-1) can be deformed to:

$$
\frac{\dot{U}_{1}}{\dot{I}_{1}}=Z_{C} \cdot t h \gamma l=Z_{X}
$$

Because of open circuit at the end of the line, then equation (1-1) can be deformed to:

$$
\frac{\dot{U}_{1}}{\dot{I}_{1}}=\frac{Z_{C}}{t h \gamma l}=X_{C}
$$

Multiplying equation (6) by equation (7), and dividing equation (6) by equation (7), can obtain line wave impedance and propagation constant:

$$
\begin{aligned}
& Z_{C}=\sqrt{Z_{X} \cdot X_{C}} \\
& \gamma=\frac{\left(\operatorname{zrcth} \sqrt{Z_{X} / X_{C}}\right)}{l}
\end{aligned}
$$

By substituting the results of (8) and (9) into (2) and (3), Multiplying equation (2) by equation (3), and dividing equation (2) by equation (3),It can be deduced as follows:

$$
\begin{gathered}
\frac{\sqrt{Z_{X} \cdot X_{C}}}{\operatorname{arcth} \sqrt{Z_{X} / X_{C}}}=\frac{1}{\left(j \omega C_{0}\right)} \\
\sqrt{Z_{X} \cdot X_{C}} \cdot \operatorname{arcth} \sqrt{Z_{X} / X_{C}}=R_{0}+j \omega L_{0}
\end{gathered}
$$

The line length $l$, the total impedance $Z_{X}$ and the total capacitive reactance $X_{C}$ in equation (10) and (11) are all known, then three parameters of $\mathrm{R}_{0}, \mathrm{~L}_{0}, \mathrm{C}_{0}$ can be calculated:

$$
\begin{aligned}
R_{0}= & \operatorname{Re}\left(\frac{\sqrt{Z_{1} \cdot Z_{2}} \cdot \operatorname{arcth} \sqrt{Z_{1} / Z_{2}}}{l}\right) \\
L_{0}= & \operatorname{Im}\left(\frac{\sqrt{Z_{1} \cdot Z_{2}} \cdot \operatorname{arcth} \sqrt{Z_{1} / Z_{2}}}{l}\right) / \omega \\
& C_{0}=\operatorname{Im}\left(\frac{\operatorname{arcth} \sqrt{Z_{1} / Z_{2}}}{l \cdot \sqrt{Z_{1} \cdot Z_{2}}}\right) / \omega
\end{aligned}
$$

\section{750kV Single Circuit Verification}

The frequency parameters of $750 \mathrm{kV}$ single-circuit transmission line are calculated respectively by the single-ended accurate calculation method, and ATP simulation proposed in this paper. The calculation results of the two methods are compared.

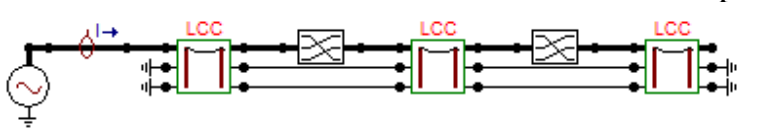

Figure1. ATPDraw circuit of $750 \mathrm{kV}$ single-circuit line

Figure 1 is a circuit model built in ATPDraw, the total length of the line is $1 \mathrm{~km}$, each section of $0.3333 \mathrm{~km}$, when the three phase line constitutes a complete transposition cycle.

First of all, the head of the line plus AC $50 \mathrm{~Hz}$, positive sequence voltage with amplitude of $10 \mathrm{~V}$, by measure the head-end current of the line under both open-circuit and short-circuit conditions; then the head of the line plus the same frequency, amplitude of the zero-sequence voltage, respectively, the first line of the current value were measured in two cases. Specific measurement results shown in Table 1, and then use the method described in this article can calculate the line frequency parameters.

Table 1: $750 \mathrm{kV}$ transmission line head-end measurement results.

\begin{tabular}{cccc}
\hline \multirow{2}{*}{ parameters } & \multirow{2}{*}{$\mathrm{U} 1 / \mathrm{V}$} & \multicolumn{2}{c}{ I1/A } \\
\cline { 3 - 4 } & & Open circuit & Short circuit \\
\hline PS & $10 \angle 0^{\circ}$ & $4.188 \mathrm{E}-5 \angle 90^{\circ}$ & $36.188 \angle-87.45^{\circ}$ \\
ZS & $10 \angle 0^{\circ}$ & $2.844 \mathrm{E}-5 \angle 90^{\circ}$ & $12.298 \angle-76.24^{\circ}$ \\
\hline
\end{tabular}

$\mathrm{PS}$ in the table indicates positive sequence, $\mathrm{ZS}$ in the table indicates zero sequence.

The left end of the line phase conductor plus AC $50 \mathrm{~Hz}$, 
amplitude of 1A positive sequence current, and the right end of the line phase grounding, then the voltage of the current source is the positive sequence impedance on the line. When the right end of the phase of the line is open, the voltage on the current source is the positive sequence tolerance of the line. If the current is zero sequence, and the other conditions remain unchanged, then the zero sequence parameters of the line also can be obtained. Because the line length takes $1 \mathrm{~km}$, the voltage value calculated by ATP is the line frequency parameter.

The calculation results of the two methods are shown in Table 2:

Table 2. Calculation results of power frequency parameters for two methods of $750 \mathrm{kV}$ transmission line.

\begin{tabular}{cccc}
\hline \multicolumn{2}{c}{ parameters } & $\begin{array}{c}\text { this paper's } \\
\text { method }\end{array}$ & ATP result \\
\hline \multirow{3}{*}{$\mathrm{PS}$} & $\mathrm{R}_{0} /(\Omega / k m)$ & 0.0122693 & 0.01227 \\
& $\mathrm{~L}_{0} /(\mathrm{H} / \mathrm{km})$ & $8.7872 \mathrm{e}-04$ & $8.79 \mathrm{e}-04$ \\
& $\mathrm{C}_{0} /(\mathrm{F} / \mathrm{km})$ & $1.33302 \mathrm{e}-08$ & $1.333 \mathrm{e}-08$ \\
\hline \multirow{3}{*}{$\mathrm{ZS}$} & $\mathrm{R}_{0} /(\Omega / \mathrm{km})$ & 0.193446 & 0.1934 \\
& $\mathrm{~L}_{0} /(\mathrm{H} / \mathrm{km})$ & $2.51399 \mathrm{e}-03$ & $2.514 \mathrm{e}-03$ \\
& $\mathrm{C}_{0} /(\mathrm{F} / \mathrm{km})$ & $9.05401 \mathrm{e}-09$ & $9.054 \mathrm{e}-09$ \\
\hline
\end{tabular}

Comparing the calculation results of this article and the results of ATP simulation software, the two calculation results are basically the same, so the calculation method is feasible.

\section{Calculation of Yu-Heng 1000 kV Line Parameters}

It is suggested that the single-ended accurate calculation by this paper should be applied to the frequency parameter calculation of UHV transmission line. The test was conducted on a section of double-circuit line with a length of $307 \mathrm{~km}$ in Yu-heng $1000 \mathrm{kV}$ UHV transmission line. It is known that the line's parameters of this section are shown in Table 3:

Table3. The parameters of Yu-Heng 1000kV line.

\begin{tabular}{cccc}
\hline \multicolumn{2}{c}{ parameters } & $\mathrm{Z} 1 / \Omega$ & $\mathrm{Z} 2 / \Omega$ \\
\hline line I & PS & $84.199 \angle 88.038^{\circ}$ & $703.6805 \angle 90^{\circ}$ \\
& $\mathrm{ZS}$ & $244.8 \angle 77.162^{\circ}$ & $1168.1097 \angle 90^{\circ}$ \\
\hline line II & PS & $84.429 \angle 88.066^{\circ}$ & $703.183 \angle 90^{\circ}$ \\
& ZS & $248.23 \angle 76.139^{\circ}$ & $1162.4786 \angle 90^{\circ}$ \\
\hline
\end{tabular}

The basic ideas of this paper have been understood from the previous article. The results obtained by calculation are shown in Table 4.

Table4. Yu-Heng 1000kV line frequency parameter.

\begin{tabular}{cccc}
\hline \multicolumn{2}{c}{ parameters } & line I & Line II \\
\hline \multirow{3}{*}{$\mathrm{PS}$} & $\mathrm{R}_{0} /(\Omega / \mathrm{km})$ & 0.0087 & 0.0086 \\
& $\mathrm{~L}_{0} /(\mathrm{H} / \mathrm{km})$ & $8.400 \mathrm{e}-04$ & $8.423 \mathrm{e}-04$ \\
& $\mathrm{C}_{0} /(\mathrm{F} / \mathrm{km})$ & $1.418 \mathrm{e}-08$ & $1.419 \mathrm{e}-08$ \\
\hline \multirow{2}{*}{$\mathrm{ZS}$} & $\mathrm{R}_{0} /(\Omega / \mathrm{km})$ & 0.1567 & 0.1710 \\
& $\mathrm{~L}_{0} /(\mathrm{H} / \mathrm{km})$ & $2.331 \mathrm{e}-03$ & $2.352 \mathrm{e}-03$
\end{tabular}

$$
\mathrm{C}_{0} /(\mathrm{F} / \mathrm{km}) \quad 8.334 \mathrm{e}-09 \quad 8.367 \mathrm{e}-09
$$

The calculation results in this line with the actual project requirements.

Now using the conventional rough calculation method, it is able to calculate the power frequency parameters of Yu-Heng I line. Table 5 is the percentage error of the calculation by the conventional method compared to the method. Figure 2 and figure 3 shows the error curves of the positive sequence and zero sequence frequency parameters of Yu-Heng I line in the conventional method, when the horizontal axis shows the length of transmission line and the vertical axis shows the error of routine calculation.

Table 5. Yu-Heng II line frequency parameters of the conventional calculation error.

\begin{tabular}{ccccccc}
\multirow{2}{*}{$1 /(\mathrm{km})$} & \multicolumn{2}{c}{$\Delta \mathrm{R}_{0} / \%$} & \multicolumn{2}{c}{$\Delta \mathrm{L}_{0} / \%$} & \multicolumn{2}{c}{$\Delta \mathrm{C}_{0} / \%$} \\
\cline { 2 - 7 } & PS & ZS & PS & ZS & PS & ZS \\
\hline 1 & $8 \mathrm{e}-5$ & $1 \mathrm{e}-4$ & $4 \mathrm{e}-5$ & $6 \mathrm{e}-5$ & $4 \mathrm{e}-5$ & $6 \mathrm{e}-5$ \\
10 & 0.00 & 0.01 & 0.00 & 0.00 & 0.00 & 0.00 \\
50 & 0.19 & 0.32 & 0.09 & 0.15 & 0.09 & 0.16 \\
100 & 0.79 & 1.29 & 0.39 & 0.61 & 0.39 & 0.64 \\
150 & 1.79 & 2.95 & 0.89 & 1.39 & 0.89 & 1.46 \\
200 & 3.23 & 5.35 & 1.60 & 2.50 & 1.60 & 2.63 \\
250 & 5.14 & 8.59 & 2.52 & 3.98 & 2.53 & 4.19 \\
300 & 7.55 & 12.7 & 3.69 & 5.85 & 3.69 & 6.17 \\
\hline
\end{tabular}

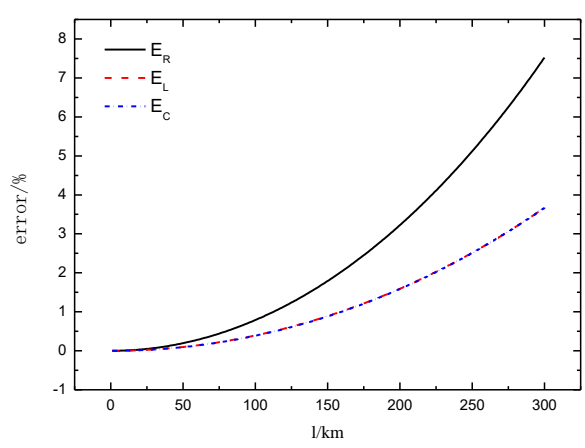

Figure2. Conventional method to calculate $1000 \mathrm{kV}$ line positive sequence frequency parameter error

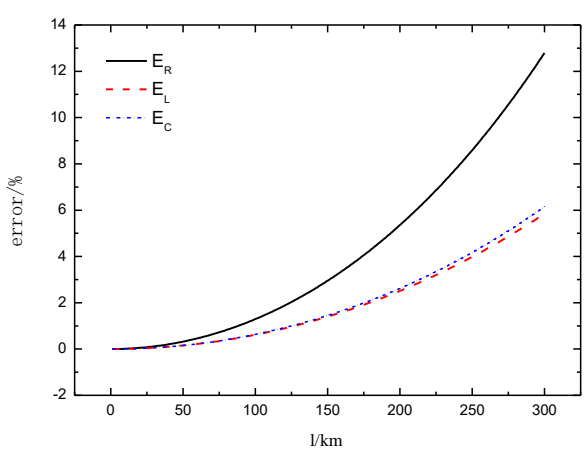

Figure3. Common method to calculate $1000 \mathrm{kV}$ line zero sequence frequency parameter error

The results show that with the increase of the line length, the error of calculating the power frequency 
parameters of the UHV transmission line using the conventional rough calculation method also increases. When the line length reaches $300 \mathrm{~km}$, the minimum error reaches $3.68 \%$. The maximum error is $12.795 \%$, which is not allowed in the project.

In summary, the conventional method for calculating long-distance UHV power transmission line frequency parameters there is a big error, but the method proposed in this article is more accurate. The $1000 \mathrm{kV}$ transmission line frequency parameters obtained by the method is enough to meet the actual project requirements.

\section{Conclusion}

1) Based on the long-line equation, the total impedance and total capacitance of the line obtained by the ratio of the voltage and the current at the head of the line which are obtained by the short circuit and the open-circuit, and then the power frequency parameters of the line are obtained. Compared with the existing single-ended method, this method does not adopt approximation and does not need to correct the calculation results to meet the engineering needs. Compared with the existing double-ended method, the proposed method does not need to measure the voltage and current of the head and ends. Simultaneously, also eliminating the need to convert different frequency parameters into frequency sequence parameters, the result is also accurate enough.

2) Calculating the power frequency parameters of Yu-Heng I and II lines of $1000 \mathrm{kV}$ UHV transmission lines by this method, the results are accurate and fully meet the engineering needs.

\section{References}

1. Shi Rong,ZhengBin,ShangYong,etal. Correction of frequency parameters for Chinas first $750 \mathrm{kV}$ double-circuit transmission lines on the same tower[J].Power System and Clean Energy,2010,26(9):29-33(in Chinese).

2. LU Ming,NINGYuhong,MAFuyu.Measuring and analysing of the power frequency parameter for power transmission line[J].High Voltage Apparatus,2004,40(3): 218-226.

3. WEI Xiaoping.The testing method and calculation of power line parameter[J].Shanxi Electric Power,2002(4):7-10.

4. DENG Chun,YUANYichao,WANGZheng,etal.Data treating in measuring power frequency parameters at long distance transmission line[J].North China Electric Power, 2004(2):48-50.

5. FU Zhong,CHENWeijiang,etal.Measurement and Calculation of Power Frequency Sequence Parameters of $1000 \mathrm{kV}$ UHVAC Double-circuit Transmission Lines on the Same Tower[J].High Voltage Engineering,2015,41(4):1285-1291. 